\title{
Influence of Forchheimer's nonlinearity and transient effects on pulse propagation in air saturated rigid granular materials
}

\author{
Diego Turo ${ }^{\text {a) }}$ \\ Department of Bioengineering, George Mason University, Fairfax, Virginia 22030 \\ Olga Umnova \\ Acoustics Research Centre, University of Salford, Salford, Greater Manchester, United Kingdom
}

(Received 14 October 2012; revised 9 June 2013; accepted 24 June 2013)

\begin{abstract}
It has been shown in the earlier work of Umnova et al. [Noise Control Eng. 50, 204-210 (2002)] that interaction of a relatively long, high amplitude acoustic pulse with a rigid porous material can be accurately described accounting for the Forchheimer nonlinearity. In the present study, the goal is to determine whether the accuracy of the modeling can be improved in the case of a lower amplitude and a shorter pulse. A model that accounts for the Forchheimer's nonlinearity and the transient effects is developed. It is assumed that all the contributions to the viscous force are additive in the time domain. The governing equations are solved numerically using finite difference time domain scheme. The results are compared with the data for two granular materials. The latter are obtained in an impedance tube and in a shock tube experiments, where acoustic pulses with various amplitudes and durations are generated. (C) 2013 Acoustical Society of America.
\end{abstract}

[http://dx.doi.org/10.1121/1.4824969]

PACS number(s): 43.25.Cb, 43.25.Jh, 43.25.Ed, 43.20.El [KVH] Pages: 4763-4774

\section{INTRODUCTION}

Peak amplitudes of shock pulses, due to explosions or those from a jet engine, can be higher than $150 \mathrm{~dB}$ and depend strongly on the distance to the receiver. The impulsive noise can be attenuated using inexpensive and mechanically robust natural porous materials, such as gravel, sand, pebbles, porous asphalt, and concrete. At high sound pressure levels, many porous materials exhibit substantial nonlinearity. This means that their acoustic properties vary with the amplitude of sound. In this regime, there is a strong interaction between different spectral components of the acoustic signal within the material. For this reason, any models formulated in the frequency domain are difficult to use for predicting sound attenuation.

A significant volume of literature is devoted to time domain numerical studies of high amplitude pulse propagation in porous materials assuming Forchheimer's filtration law. ${ }^{1}$ The latter accounts for a linear growth of static flow resistivity with the increase of flow velocity. In the presence of sound, the same type of flow resistivity dependence on particle velocity amplitude is assumed. In Ref. 2, for instance, the propagation of a moderate amplitude acoustic pulse in a rigid porous material has been studied, assuming Forchheimer's correction to Darcy's law and quadratic nonlinearity. This approximation is valid when most of the acoustic energy is concentrated in the lower frequency range, which implies relatively long pulses.

For shorter pulses, the transient (also called memory or inertial) effects need to be taken into consideration. They account for a non-instantaneous response of the medium to

\footnotetext{
a) Author to whom correspondence should be addressed. Electronic mail: diegoturo@gmail.com
}

sound. Modeling of the transient effects in a linear regime has been performed in the past and is described in Refs. 3-5. The analysis given in Refs. 4 and 5 is valid for short pulses of low amplitude and with most of the energy concentrated in the higher frequency range. Accounting for the transient effects is also important for predicting sound attenuation in concentrated emulsions. $^{6}$

In this work, the interaction of an intermediate amplitude and duration pulse with rigid frame porous materials is studied. The contributions of both Forchheimer's nonlinearity and the transient effects to the drag force are taken into account, in order to correctly predict the material response.

In Sec. II the approach to modeling is described. The linear model is formulated first. It is assumed that all the contributions to the drag force are additive in the time domain. This is strictly valid for the drag force acting, for instance, on a sphere moving arbitrarily in a viscous incompressible fluid. ${ }^{7}$ However, it is argued that similar expression can be formulated for the force arising when acoustic pulse propagates in a rigid porous material with an arbitrary pore geometry. It is shown that when transferred into frequency domain, the expression satisfies physically correct low and high frequency limits. Forchheimer's nonlinearity term is then incorporated into the time domain model to account for the finite amplitude effects.

The governing equations are solved numerically using a finite difference time domain (FDTD) scheme. The numerical technique described in Refs. 8 and 9 has been used before to consider the contribution of inertia, convection, viscosity, and relaxation effects. In the present work, the relative importance of viscous and transient effects is evaluated using two numerical codes. One of them accounts for the viscosity effects (including Forchheimer's nonlinear term) only, while the other accounts for both viscosity and transient effects. 
The range of acoustic pulse and material parameters, where both Forchheimer's nonlinearity and transient effects contributions are strong, is identified in Sec. III.

In Secs. IV and V the experiments are described. Predictions of the linear model are compared with the impedance tube measurements. The shock tube setup was used to generate acoustic pulses with amplitudes between $2 \mathrm{kPa}$ and $20 \mathrm{kPa}$ and durations of $3 \mathrm{~ms}$ and $1.5 \mathrm{~ms}$. The nonlinear model has been validated by comparing its results with the shock tube data. The main findings are summarized in Sec. VI.

\section{THE MODEL}

The response of a rigid frame porous material to an acoustic excitation is often described using complex tortuosity ${ }^{10}$ and complex compressibility functions. ${ }^{11,12}$ The former accounts for the viscosity related effects, whereas the latter is designed to account for thermal losses. For low frequency sound the viscous losses are described by a static flow resistivity $\sigma_{0}$. This range of frequencies is referred to as the viscous regime. It is assumed in the following that flow resistivity grows linearly with flow velocity

$$
\sigma(U) \approx \sigma_{0}(1+\xi|U|),
$$

where $\xi$ is Forchheimer's nonlinearity parameter. Both $\sigma_{0}$ and $\xi$ can be measured in standard flow resistivity tests. At higher frequencies, the response is influenced by the temporal delay in viscous boundary layer development. This range of frequencies is referred to as the inertial or the transient regime. Material response in this regime is governed by an additional parameter, $\Lambda$, which is called the characteristic viscous length. ${ }^{10}$

We assume that the terms describing viscous and transient regimes are additive in the expression for the complex tortuosity function

$$
\alpha(\omega)=\alpha_{\infty}+\frac{\sigma_{0} \phi}{-i \omega \rho_{0}}+\frac{2 \alpha_{\infty}}{\Lambda} \sqrt{\frac{\eta}{-i \omega \rho_{0}}},
$$

where $\alpha_{\infty}$ is tortuosity, $\phi$ is porosity, $\omega$ is angular frequency, and $\rho_{0}$ and $\eta$ are density and dynamic viscosity of air, respectively. The function defined by Eq. (2) is different from the conventional scaling function of the equivalent fluid model. ${ }^{10}$ However, it still satisfies the same physically correct low and high frequency limits, i.e., $\alpha(\omega \rightarrow \infty)=\alpha_{\infty}$ $+2 \alpha_{\infty} \sqrt{\eta} /\left(\Lambda \sqrt{-i \omega \rho_{0}}\right) \quad$ and $\quad \alpha(\omega \rightarrow 0)=\alpha_{\infty}+\sigma_{0} \phi /$ $\left(-i \omega \rho_{0}\right)$. The scaling function in Ref. 10 has been chosen to be the simplest one that satisfies these limits. However, a whole family of other functions with the same limits can be constructed. Equation (2) describes one of them.

In the time domain, the use of expression (2), leads to the following expression for the drag force $F(t)$ acting on a unit volume of air in a porous material

$$
\begin{aligned}
F(t)= & \rho_{0}\left(\alpha_{\infty}-1\right) \frac{d v}{d t}+\sigma \phi v \\
& +\frac{2 \alpha_{\infty}}{\Lambda} \sqrt{\frac{\eta}{\pi \rho_{0}}} \int_{-\infty}^{t} \frac{d v}{d t} \frac{d \tau}{\sqrt{t-\tau}} .
\end{aligned}
$$

The structure of this expression is similar to that of the friction force $f(t)$, acting on a sphere of radius $R$, which is moving in an arbitrary manner with velocity $v$ in an unbounded incompressible fluid ${ }^{7}$

$$
\begin{aligned}
f(t)= & \frac{2}{3} \pi \rho_{0} R^{3} \frac{d v}{d t}+6 \pi \eta R v \\
& +6 \rho_{0} R^{2} \sqrt{\frac{\eta \pi}{\rho_{0}}} \int_{-\infty}^{t} \frac{d v}{d t} \frac{d \tau}{\sqrt{t-\tau}} .
\end{aligned}
$$

The second term in Eq. (4) is known as the Stokes force. The third term is known as the transient or Basset force. It takes into account the influence of the velocity history on its value at present time and is called the transient term in this work. The same name is used for the third term on the right hand side of Eq. (3).

The transient term can be neglected if the influence of the early stages of the viscous boundary layer formation is not significant. In the time domain this implies relatively long pulses. In the frequency domain, this is equivalent to the viscous regime of interaction mentioned above. In this regime the viscous boundary layer thickness exceeds the characteristic pore size. The acoustic signal has mostly low frequency content, $\omega \ll \omega_{c}$.

The transient effects become significant for shorter pulses and dominate for the shortest ones. In the frequency domain, this is equivalent to the assumption of the transient regime mentioned above. The viscous boundary layer thickness is much less than the characteristic pore size. The acoustic signal has mostly high frequency content, $\omega \gg \omega_{c}$. Here $\omega_{c}$ is the critical angular frequency of the medium defined as

$$
\omega_{c}=\frac{\sigma_{0}^{2} \phi^{2} \Lambda^{2}}{4 \eta \rho_{0} \alpha_{\infty}^{2}} .
$$

The use of a scaling function, similar to that described by Eq. (2), results in the following expression for complex compressibility:

$$
\begin{aligned}
C(\omega)= & \gamma-(\gamma-1) \\
& \times\left(1+\frac{\phi \eta}{-i \omega N_{\operatorname{Pr}} \rho_{0} k^{\prime}}+\frac{2}{\Lambda^{\prime}} \sqrt{\frac{\eta}{-i \omega N_{\operatorname{Pr}} \rho_{0}}}\right)^{-1},
\end{aligned}
$$

where $N_{\operatorname{Pr}}$ is Prandtl number $\left(N_{\operatorname{Pr}}=c_{p} \eta / \kappa, c_{p}\right.$ is specific heat at constant pressure and $\kappa$ is thermal conductivity), $\gamma$ is adiabatic constant, $\Lambda^{\prime}$ is characteristic thermal length, and $k^{\prime}$ is thermal permeability. This function has the same low and high frequency limits as the one introduced in Refs. 11 and 12.

The condition $\omega \gg \omega_{c}$ has been always met in the experiments described in this work. For this reason, the high frequency limit of complex compressibility (6), i.e.,

$$
C(\omega)=1+\frac{2(\gamma-1)}{\Lambda^{\prime}} \sqrt{\frac{\eta}{-i \omega N_{\operatorname{Pr}} \rho_{0}}},
$$

has been used in the model. 
In the frequency domain the linear governing equations are

$$
\begin{aligned}
& -i \omega \alpha(\omega) \rho_{0} U=-\phi \nabla P, \\
& -i \omega C(\omega) P=-\frac{\rho_{0} c^{2}}{\phi} \nabla \cdot U,
\end{aligned}
$$

where $c$ is sound speed in air, $U$ is particle velocity averaged over the porous material volume, and $P$ is acoustic pressure.

First, the Fourier transformation of Eqs. (8) and (9) is performed, where expression (2) is used for the complex tortuosity function and complex compressibility function is described by Eq. (7). After that, Forchheimer's correction to Darcy's law (1) is introduced into the time domain equations. This results in the following equations:

$$
\begin{aligned}
& \alpha_{\infty} \rho_{0} \frac{\partial v}{\partial t}+\frac{2 \alpha_{\infty} \rho_{0}}{\Lambda} \sqrt{\frac{\eta}{\pi \rho_{0}}} \int_{-\infty}^{t} \frac{\partial v}{\partial t^{\prime}} \frac{d t^{\prime}}{\sqrt{t-t^{\prime}}} \\
& +\sigma_{0} \phi(1+\xi|v|) v=-\phi \frac{\partial p}{\partial x}, \\
& \frac{\partial p}{\partial t}+\frac{2(\gamma-1)}{\Lambda^{\prime}} \sqrt{\frac{\eta}{N_{\mathrm{Pr}} \pi \rho_{0}}} \int_{-\infty}^{t} \frac{\partial p}{\partial t^{\prime}} \frac{d t^{\prime}}{\sqrt{t-t^{\prime}}}=-\frac{\rho_{0} c^{2}}{\phi} \frac{\partial v}{\partial x},
\end{aligned}
$$

where $p$ and $v$ are pressure and particle velocity in time domain.

If the viscous force with Forchheimer's correction [third term on the left hand side of Eq. (10)] can be neglected, in comparison with transient term (second term on the left hand side), than Eq. (10) coincides with the one used to model propagation of low amplitude short pulses in Refs. 3 and 4. When transient term is small compared to the viscous force with Forchheimer's correction, Eq. (10) is reduced to the one used to model high amplitude long pulse propagation in Ref. 2.

\section{ANALYSIS OF DIMENSIONLESS GOVERNING EQUATION}

In order to define the range of parameters, where the contributions of the transient term and the Forchheimer's term are equally strong, an analysis of coefficients in Eqs. (10) and (11) is performed.

First, the following dimensionless quantities are introduced: $p^{*}=p / p_{\text {ref }}, v^{*}=v / v_{\text {ref }}, t^{*}=t / t_{\text {ref }}$, and $x^{*}=x / x_{\text {ref }}$. The reference values for the pressure, particle velocity, time, and space coordinates have been chosen as follows: $p_{\text {ref }}=\rho_{0} c v_{\text {ref }}=p_{\text {exc }}, v_{\text {ref }}=p_{\text {exc }} /\left(\rho_{0} c\right), t_{\text {ref }}=t_{\text {exc }}$, and $x_{\text {ref }}$ $=v_{\text {ref }} t_{\text {exc }}=t_{\text {exc }} p_{\text {exc }} /\left(\rho_{0} c\right)$. Here $p_{\text {exc }}$ is pressure amplitude and $t_{\mathrm{exc}}$ is duration of the excitation pulse.

Second, Eqs. (10) and (11) are written in the dimensionless form

$$
\begin{aligned}
& \frac{\partial v^{*}}{\partial t^{*}}+\frac{2}{\Lambda} \sqrt{\frac{\eta t_{\mathrm{exc}}}{\pi \rho_{0}}} \int_{-\infty}^{t} \frac{\partial v^{*}}{\partial t^{* \prime}} \frac{d t^{* \prime}}{\sqrt{t^{*}-t^{* \prime}}} \\
& \quad+\frac{\sigma_{0} \phi t_{\mathrm{exc}}}{\alpha_{\infty} \rho_{0}}\left(1+\xi \frac{p_{\mathrm{exc}}}{\rho_{0} c}\left|v^{*}\right|\right) v^{*}=-\frac{\phi \rho_{0} c^{2}}{\alpha_{\infty} p_{\mathrm{exc}}} \frac{\partial p^{*}}{\partial x^{*}},
\end{aligned}
$$

$$
\frac{\partial p^{*}}{\partial t^{*}}+\frac{2(\gamma-1)}{\Lambda^{\prime}} \sqrt{\frac{\eta t_{\mathrm{exc}}}{N_{\mathrm{Pr}} \pi \rho_{0}}} \int_{-\infty}^{t} \frac{\partial p^{*}}{\partial t^{* \prime}} \frac{d t^{* \prime}}{\sqrt{t^{*}-t^{* \prime}}}=-\frac{\rho_{0} c^{2}}{\phi p_{\mathrm{ref}}} \frac{\partial v^{*}}{\partial x^{*}} .
$$

After separation the linear viscous term from the quadratic one (Forchheimer's term), Eq. (12) becomes

$$
\begin{aligned}
& \frac{\partial v^{*}}{\partial t^{*}}+\sqrt{\frac{4 \eta t_{\mathrm{exc}}}{\pi \Lambda^{2} \rho_{0}}} \int_{-\infty}^{t} \frac{\partial v^{*}}{\partial t^{* \prime}} \frac{d t^{* \prime}}{\sqrt{t^{*}-t^{* \prime}}} \\
& +\frac{\sigma_{0} \phi t_{\mathrm{exc}}}{\alpha_{\infty} \rho_{0}} v^{*}+\xi \frac{\sigma_{0} \phi p_{\mathrm{exc}} t_{\mathrm{exc}}}{\alpha_{\infty} \rho_{0}{ }^{2} c} v^{* 2}=-\frac{\phi \rho_{0} c^{2}}{\alpha_{\infty} p_{\mathrm{exc}}} \frac{\partial p^{*}}{\partial x^{*}} .
\end{aligned}
$$

The transient term has the same order of magnitude as the linear viscous term if the following condition is satisfied:

$$
\sqrt{\frac{4 \eta}{\pi \Lambda^{2} \rho_{0}} t_{\mathrm{exc}}} \approx \frac{\sigma_{0} \phi}{\alpha_{\infty} \rho_{0}} t_{\mathrm{exc}} .
$$

This leads to the following condition on pulse duration:

$$
t_{\mathrm{exc}} \approx\left(\frac{1}{\pi}\right) \frac{4 \alpha_{\infty}^{2} \rho_{0} \eta}{\sigma_{0}^{2} \phi^{2} \Lambda^{2}} .
$$

Introducing the critical frequency (5), Eq. (16) can be rewritten as

$$
t_{\mathrm{exc}} \approx \frac{1}{\pi \omega_{c}} .
$$

From the frequency domain analysis, ${ }^{13}$ it was found that the transient and the linear viscosity effects are comparable when the angular frequency of sound is equal to the critical frequency defined by Eq. (5). Equation (17) is the time domain analog of this condition.

Transient effects are stronger than those of the linear viscosity if

$$
t_{\mathrm{exc}}<\frac{1}{\pi \omega_{c}} .
$$

Now the coefficients at the transient and Forchheimer's terms are compared. They are the same order of magnitude when

$$
\sqrt{\frac{4 \eta}{\pi \Lambda^{2} \rho_{0}} t_{\mathrm{exc}}} \approx \xi \frac{\sigma_{0} \phi}{\alpha_{\infty}} \frac{p_{\mathrm{exc}}}{\rho_{0}^{2} c} t_{\mathrm{exc}}
$$

which means

$$
t_{\mathrm{exc}} \frac{p_{\mathrm{exc}}{ }^{2}}{\rho_{0} c^{2}} \approx\left(\frac{\rho_{0}}{\xi^{2} \pi}\right) \frac{4 \alpha_{\infty}^{2} \rho_{0} \eta}{\sigma_{0}^{2} \phi^{2} \Lambda^{2}}=\left(\frac{\rho_{0}}{\xi^{2}}\right) \frac{1}{\pi \omega_{c}} .
$$

Combining conditions (17) and (20), we conclude that transient, linear and Forchheimer's terms have same order of magnitude when pressure amplitude satisfies the following condition:

$$
t_{\mathrm{exc}} \frac{p_{\mathrm{exc}}^{2}}{\rho_{0} c^{2}} \approx\left(\frac{\rho_{0}}{\xi^{2}}\right) \frac{1}{\pi \omega_{c}} \Rightarrow p_{\mathrm{exc}} \approx \frac{\rho_{0} c}{\xi} .
$$




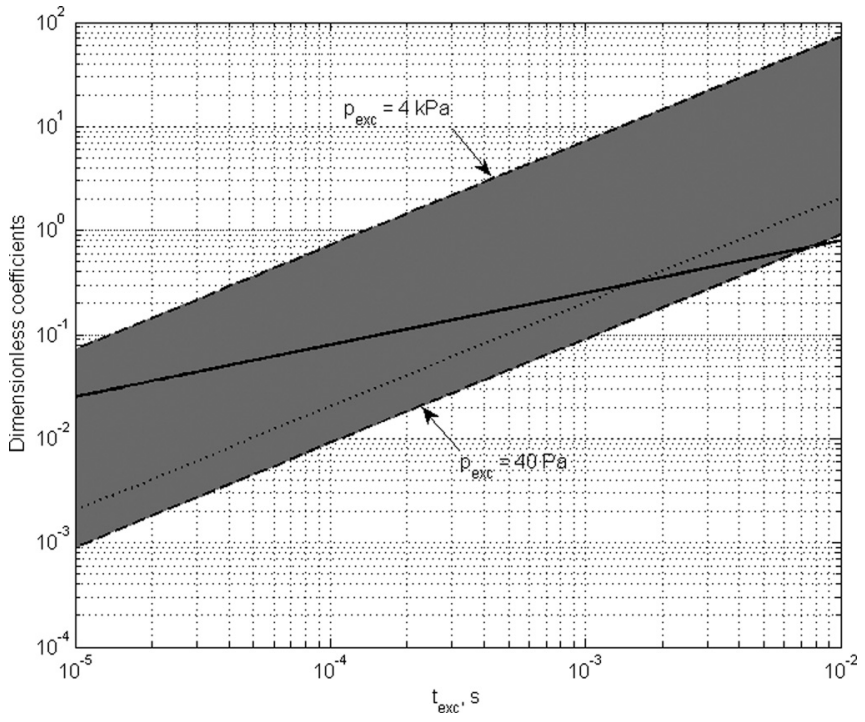

FIG. 1. Dimensionless coefficients in Eq. (14) for different pulse durations and amplitudes. Lead shot (parameters in Table I). Solid line: transient term coefficient; dashed lines: quadratic viscous (Forchheimer nonlinearity) term coefficient for different pulse amplitudes; dotted line: linear viscous term coefficient.

The transient term is dominant, compared to the Forchheimer's term, if

$$
t_{\mathrm{exc}}<\frac{1}{p_{\mathrm{exc}}{ }^{2}}\left(\frac{\rho_{0} c}{\xi}\right)^{2} \frac{1}{\pi \omega_{c}} .
$$

Rearranging Eq. (20), we conclude that Forchheimer's term and the transient term are the same order of magnitude when

$$
p_{\mathrm{exc}} \approx \frac{\rho_{0} c}{\xi} \sqrt{\frac{1}{\pi \omega_{c} t_{\mathrm{exc}}}} .
$$

For the pulse with amplitude higher than that estimated by Eq. (23), Forchheimer's nonlinearity effect is dominant. For the pulses with lower amplitude or with durations defined by inequality (22), the transient effect is stronger.

Typical values of Forchheimer's nonlinearity parameter $\xi$ are in the range $\{0,10\} \mathrm{s} / \mathrm{m} .^{2,14}$ For materials with $\xi$ $\ll 1 \mathrm{~s} / \mathrm{m}$ (such as mineral wool or granular material with very small particles, e.g., sand), the three effects are comparable in strength only at high level of excitation. For instance, for $\xi=0.1 \mathrm{~s} / \mathrm{m}$ acoustic pulse amplitude predicted by Eq. (21) is around $4 \mathrm{kPa}$. For more common granular media (such as pebbles, stones, and asphalt) Forchheimer's parameter $\xi$ values are of the order of unity. This means that only for pressure amplitude around $0.4 \mathrm{kPa}$ and duration defined by Eq. (17) the linear viscosity, Forchheimer's nonlinearity and the transient effects are comparable in strength.
In Fig. 1 the dimensionless coefficient values are shown as functions of the pulse duration for two excitation amplitudes. The calculations have been performed for the lead shot with parameters summarized in Table I. The viscous and Forchheimer's nonlinearity parameters were measured, tortuosity and porosity were reported in Refs. 13 and 14, whereas thermal parameters have been evaluated using the cell model. ${ }^{15}$ In Table I $f_{c}=\omega_{c} /(2 \pi)$ is the critical frequency of the sample.

The transient term has the same order of magnitude as the linear viscous term for pulses with time durations on the order of $1.5 \mathrm{~ms}$. The effect of the Forchheimer's nonlinearity is weak for a pulse with amplitude of $40 \mathrm{kPa}$, while for that with amplitude of $4 \mathrm{kPa}$ it dominates over the viscous and the inertial effects irrespective of pulse duration.

\section{VALIDATION OF THE LINEAR MODEL: IMPEDANCE TUBE EXPERIMENTS}

The aim of this part of the work is to validate the model for a low amplitude pulse and to investigate the relative importance of viscous, inertial, and Forchheimer's nonlinearity effects. The data are compared with the model predictions obtained (a) accounting for Forchheimer' correction, (b) without it, i.e., setting $\xi=0$ in Eq. (10), (c) accounting for transient effect and (d) neglecting the transient term in Eq. (10).

A FDTD method with one-dimensional staggered spatial scheme is used to numerically solve Eqs. (10) and (11). The two-dimensional representation of this scheme is illustrated in Ref. 16. The stability condition requires the Courant number to be smaller than unity. ${ }^{17}$

The experiments and numerical calculations have been performed for a packing of gravel. Its parameters are summarized in Table I.

A low amplitude acoustic pulse is generated in an impedance tube. The tube is designed specifically for investigating acoustic pulse propagation in granular media. ${ }^{18}$ The incident pulse is generated by a loudspeaker, then reflected by a $5.5 \mathrm{~cm}$ thick sample of gravel placed $4.5 \mathrm{~cm}$ away from the rigid wall and recorded by a microphone placed at $0.079 \mathrm{~m}$ from the sample surface. This arrangement is used to maximize the influence of the transient effects.

The gravel used in the experiments is identical to that described in Ref. 13, however, its static flow resistivity and Forchheimer's nonlinearity parameter were measured again. Although the flow resistivity and Forchheimer's parameter of gravel are close to that of the lead shot, the characteristic viscous length is substantially smaller. This means that the influence of a transient term is stronger in this material.

The measured spectrum of the pulse is shown in Fig. 2. The proportion of the pulse energy distributed above the critical frequency $f_{c}$ is $99.8 \%$, so the inertial regime is achieved.

\begin{tabular}{|c|c|c|c|c|c|c|c|c|c|}
\hline Sample & Particle radius $\left(10^{-3} \mathrm{~m}\right)$ & $\alpha_{\infty}$ & $\phi$ & $\sigma_{0}\left(\mathrm{Pas} / \mathrm{m}^{2}\right)$ & $\xi(\mathrm{s} / \mathrm{m})$ & $\Lambda\left(10^{-3} \mathrm{~m}\right)$ & $\Lambda^{\prime}\left(10^{-3} \mathrm{~m}\right)$ & $k_{0}^{\prime}\left(10^{-9} \mathrm{~m}^{2}\right)$ & $f_{c}(\mathrm{~Hz})$ \\
\hline Lead shot & 1.89 & 1.60 & 0.385 & 1026 & 3.70 & 0.55 & 0.79 & 31.8 & 33.18 \\
\hline Gravel & 4.51 & 1.55 & 0.380 & 1064 & 4.06 & 0.19 & 1.84 & 173.0 & 10.60 \\
\hline
\end{tabular}

TABLE I. Samples parameters: lead shot and gravel. 


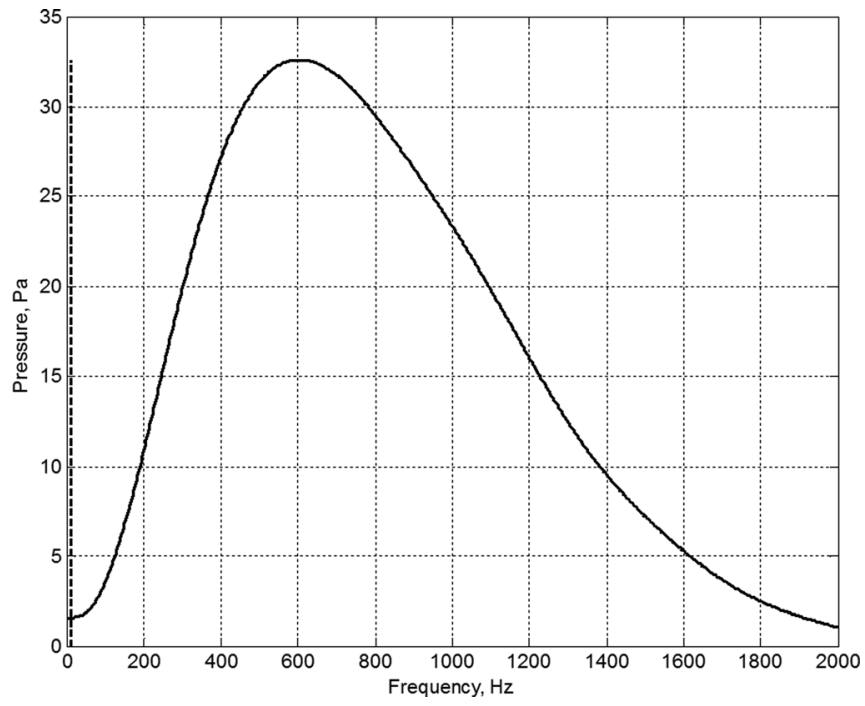

FIG. 2. Spectrum of a low amplitude (48 Pa) pulse. Dashed line: critical frequency of gravel (data in Table I).

Figures 3(a)-3(c) shows that, in this case, the model predictions are in a very good agreement with the reflection data. Prediction accounting for Forchheimer's correction is almost undistinguishable from that without it [Fig. 3(b)]. Accounting for the transient effect leads to a better agreement with the data [Fig. 3(c)].

\section{VALIDATION OF THE NONLINEAR MODEL: SHOCK TUBE EXPERIMENTS}

The aim of this part of the work is to validate the nonlinear model and to investigate the importance of the transient effects in this case.

A shock tube set up can be used to generate pulses with amplitudes up to $65 \mathrm{kPa}$ and durations longer than $1.6 \mathrm{~ms} .{ }^{19}$ A shock tube is an enclosed metal pipe with a chamber at one end and a rigid wall at the other one. A sample can be placed at the end of the pipe or in the middle of it. A membrane separates the chamber from the rest of the pipe. The chamber is pressurized until the break of the membrane. When the membrane breaks down, a shock wave propagates along the tube and the microphones placed in front and at the back of the sample record incident, reflected, and transmitted pulses. With this setup, the pulse amplitude and duration can be controlled in two ways: varying the type of membrane or using multiple reflections of the pulse from the pipe ends. The former can be achieved using membranes made from different materials (the stiffer the material, the higher the pulse amplitude) or with different thicknesses (the thicker the membrane, the higher the pulse amplitude). The latter, instead, uses the deformation of the shock wave due to its nonlinear propagation in air and attenuation due to the sample. The shock deformation due to the air nonlinearity changes both amplitude and pulse duration.

A pulse with maximum amplitude of $25 \mathrm{kPa}$ and duration of $2.5 \mathrm{~ms}$ is generated rupturing the aluminum foil membrane. A sketch of the experimental setups is shown in Fig. 4.

Piezoelectric microphones record pressure at fixed positions along the tube. In the case of a reflection setup,
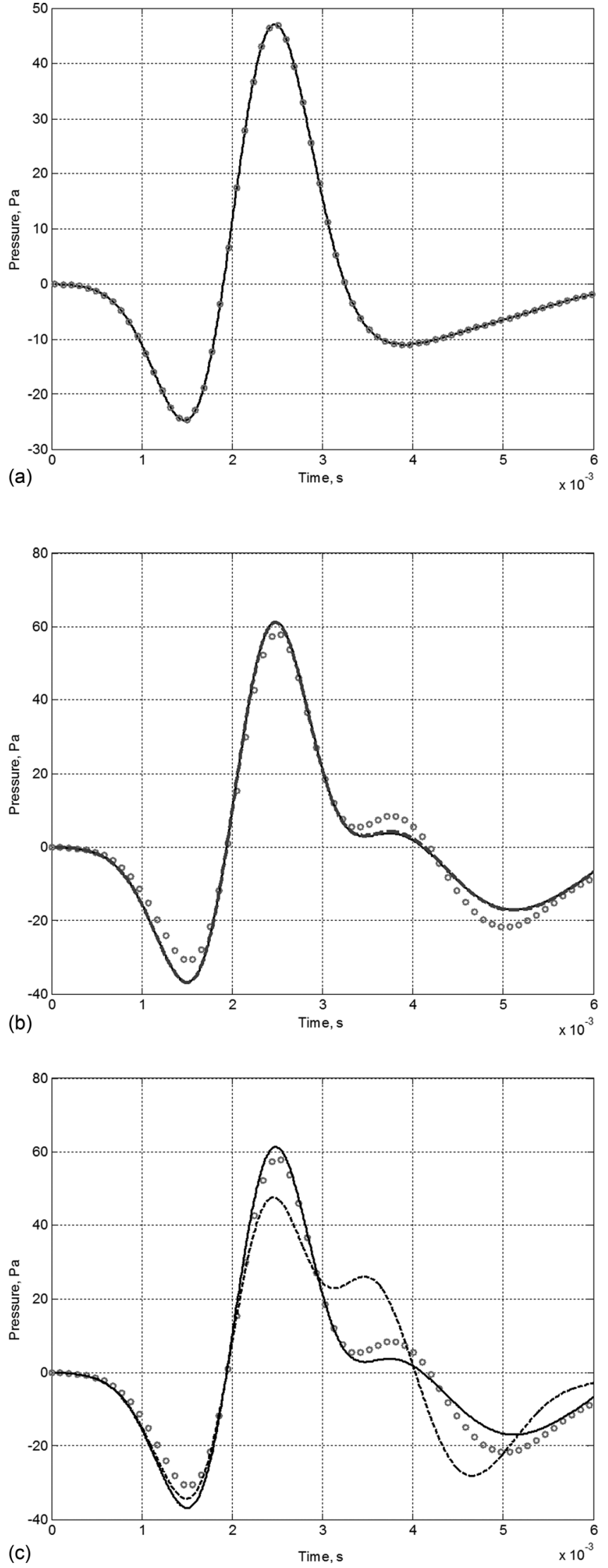

FIG. 3. Low amplitude pulse reflection. (a) Incident pulse. Gray circles: data; solid line: data reconstruction. Pulse reflected by $5.5 \mathrm{~cm}$ layer of gravel, air gap $4.5 \mathrm{~cm}$. (b) Gray circles: data; solid line: Eqs. (10) and (11); dashed gray line equations (10) and (11) with $\xi=0$. (c) Gray circles: data; solid line: Eqs. (10) and (11); dashed line Eqs. (10) and (11) without transient term in Eq. (10). 


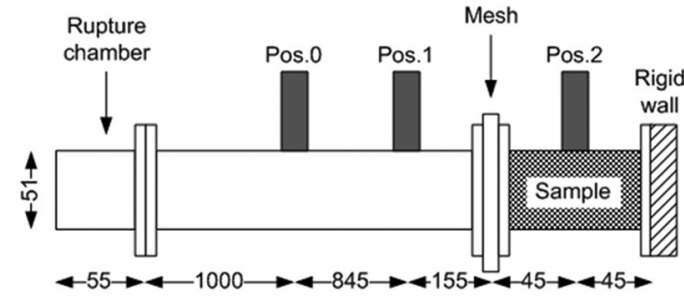

(a)

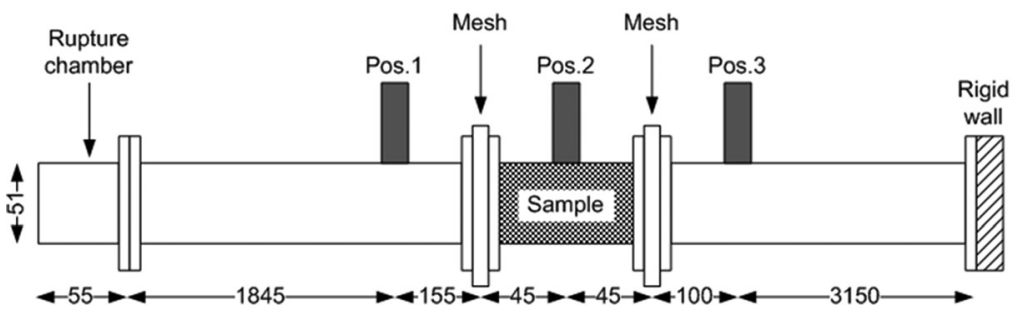

FIG. 4. Shock tube setup for reflection (a) and transmission (b) measurements. All dimensions are shown in $\mathrm{mm}$.
Fig. 4(a), the sample is placed at the end of the tube and records of the incident pulse and the reflected pulse are taken. The sample holder has a microphone holder placed at its middle point, which allows recording pulses propagating through the sample.

In the case of a transmission setup, Fig. 4(b), a tube extension of $3.25 \mathrm{~m}$ is used and the sample holder is placed between the main tube and the extension. To avoid the superposition of the pulse transmitted through the sample and the pulse reflected from the rigid termination of the tube, the shock front time of flight must be at least twice the pulse duration. With an extension of $3.25 \mathrm{~m}$, a time of flight is around $19 \mathrm{~ms}[t=2(3.25 / c) \mathrm{s}]$. This is around 6 times longer than the pulse duration, so the superposition between the two pulses is avoided.

A proportion of the pulse energy which corresponds to frequencies lower than $1 \mathrm{kHz}$ is $97 \%$ (Fig. 7). This means that the shortest wavelength of interest is $34.3 \mathrm{~cm}$, which is around 6 times longer than the tube diameter $(5.7 \mathrm{~cm})$. This confirms the validity of the plane wave approximation. For this reason a one dimensional numerical scheme has been implemented to solve the equations.

The peak pressure is measured by a pressure transducer at a distance of $1 \mathrm{~m}$ from the foil, Pos. 0 in Fig. 4(a). The pressure is also measured at different locations along the tube with three piezoelectric transducers PCB Piezotronics113B21. Each transducer can measure pressures up to $7000 \mathrm{kPa}$ (70 bar). The measurements confirm that the peak pressure does not vary significantly from one rupture to another. However, to take into account the small variations due the foil mounting procedure, the value averaged over four measurements is used for comparison with the model.

\section{A. Pulse propagation in air}

The influence of the nonlinear convection is neglected in the model. In air, as shown below, this is possible due to relatively low pulse amplitude and short distances between the transducers.

Convection leads to attenuation of the pulse peak and the increase of its duration. In Fig. 5 the distortions due to nonlinear convection are shown for a pulse with amplitude of around $18 \mathrm{kPa}$. The predictions have been obtained solving the Burgers' equation ${ }^{20}$

$$
\frac{\partial p}{\partial x}=\frac{\beta}{\rho_{0} c} p \frac{\partial p}{\partial t^{\prime}}+\frac{\varepsilon}{2 c^{3}} \frac{\partial^{2} p}{\partial t^{\prime 2}},
$$

using a FDTD scheme. Here $t^{\prime}=t-x / c, \beta=(\gamma+1) / 2$ is coefficient of nonlinearity, and $\varepsilon$ is the diffusivity coefficient which accounts for heat conduction and viscosity of air.

A one dimensional MacCormack scheme ${ }^{21}$ was implemented. A forward difference predictor and backward difference corrector were used for evaluating the convection term, whereas a central difference was performed for evaluating the diffusion term.

The nonlinear convection contribution is evident at Pos. 0 . The pulse amplitude is attenuated by $20 \%$ and the pulse duration is increased by $30 \%$.

These data are consistent with the numerical results and also with a simple triangular pulse approximation described

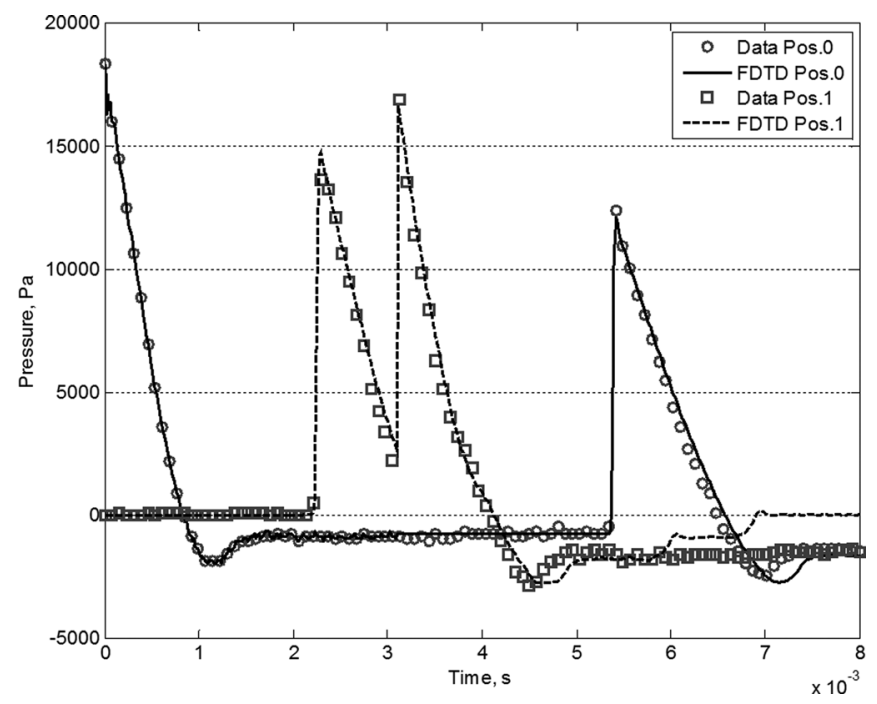

FIG. 5. Pulse propagation in an empty shock tube. Using the reflection setup with no sample, the pulse has been measured at Pos. 0 and Pos. 1 as in Fig. 4(a). The measured shock profile at Pos. 1 is affected by the pulse reflected from the rigid termination. 
in Ref. 7. According to this approximation, the amplitude $\Delta p^{\prime}$ and the pulse duration $T^{\prime}$ at a distance $x$ are calculated as

$$
\left\{\begin{array}{l}
T^{\prime}=T_{0} \sqrt{\left[1+\left(\frac{\gamma+1}{2} \frac{\Delta p_{0} x}{\rho_{0} c_{0}^{3} T_{0}}\right)\right]} \\
\Delta p^{\prime}=\frac{\Delta p_{0}}{\sqrt{\left[1+\left(\frac{\gamma+1}{2} \frac{\Delta p_{0} x}{\rho_{0} c_{0}^{3} T_{0}}\right)\right]}},
\end{array}\right.
$$

where $\Delta p_{0}, T_{0}$ are amplitude and duration of the pulse at the initial position $x=0$. In Eq. (25) the influence of the dissipation is neglected. In order to be able to use Eq. (25), the measured waveform is approximated with a triangular pulse of amplitude $18 \mathrm{kPa}$ and duration of $0.84 \mathrm{~ms}$. The distance traveled by the pulse between the first and the second recordings at Pos. 0 is $x=0.845 \mathrm{~m}$. According to Eq. (25) the peak is attenuated by $17 \%$ whereas the pulse duration (measured from a shock front to a zero pressure point) is increased by $21 \%$ in a reasonable agreement with the data. This confirms the validity of Eqs. (25).

Using these equations it can be shown, that if a pulse with amplitude $10 \mathrm{kPa}$ or less and with duration of $1 \mathrm{~ms}$ travels between Pos. 1 and Pos. $3(x=0.345 \mathrm{~m})$, its amplitude decrease and its duration increase are both less than $5 \%$ of the initial values. Due to this, convection contribution is not accounted for, when propagation between these transducer positions is considered. In the following, pulses with amplitude less than $10 \mathrm{kPa}$ are used for comparisons with the model.

It has been justified in Ref. 2 that nonlinear convection can be neglected for a pulse propagating inside porous material.

\section{B. Pulse propagation in porous materials}

In the shock tube, multiple data sets can be recorded performing a single rupture of the foil. In Fig. 6 a typical data set is shown and the main advantage of using this experimental setup is demonstrated. A single rupture allows collecting at least three sets of data. The first set includes the direct incident pulse superimposed with the one reflected by the sample surface, the pulse recorded inside the sample, and the pulse transmitted through the sample. The second set of pulses is the same as the first one with the difference that the pulse reflected by the rigid wall of the chamber now acts as the incident one. This set of pulses is separated from the first one by $0.012 \mathrm{~s}$. This is the time required for the pulse to propagate over $4.1 \mathrm{~m}$. This distance is equal to twice the distance between Pos. 1 and the wall of the chamber plus twice the distance between Pos. 1 and the surface of the sample. Third set of pulses is initiated by the pulse reflected from the rigid termination of the extension. In fact, the order of appearance of records is inverted in this set. The fourth set of pulses is initiated by the reflection of the second set from the chamber wall. The delay between the first and the second sets is the same as that between the second and the fourth. Due to these multiple reflections, it is possible to collect the data sets with incident pulses of different amplitudes and durations.

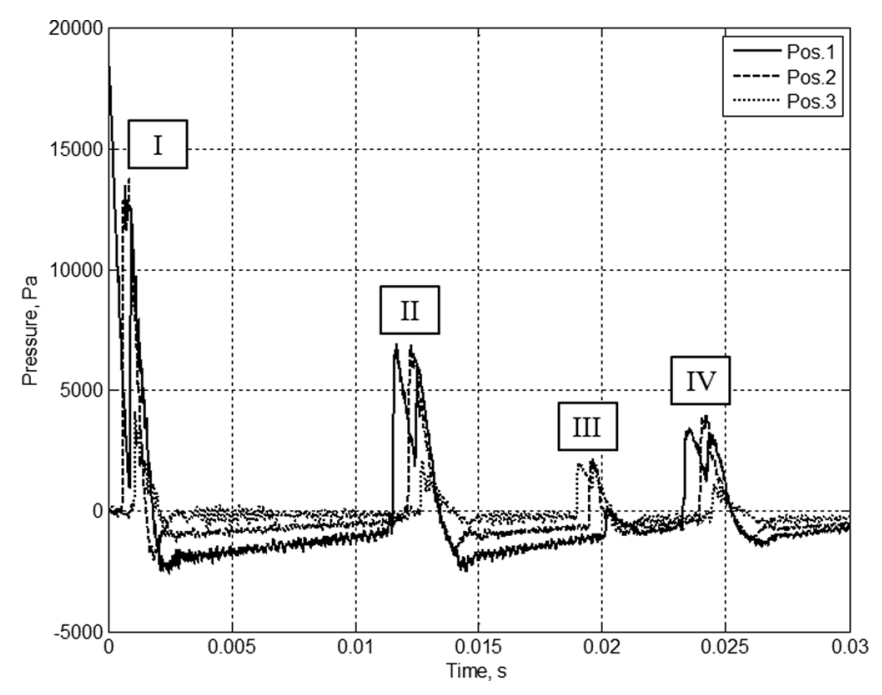

FIG. 6. Shock tube measurement: transmission setup. Data obtained from a single foil rupture. Record positions are shown in Fig. 4(b). The sample holder is filled with the lead shot.Set of data I: incident pulse superimposed with the one reflected by the sample surface (solid line); pulse recorded inside the sample (dashed line); transmitted pulse (dotted line). Set of data II: same as I, but the pulse reflected from the rigid termination of the chamber acts as an incident one. Set of data III is due to the pulse reflected by the rigid wall of the extension. Set of pulses IV is the reflection of set II from the chamber wall

A FDTD method is used to numerically solve Eqs. (10) and (11). In order to use the convolution integral approximation suggested in Ref. 13, the time step is set to a value two orders of magnitude smaller than the shock front thickness.

The pulses used in computations satisfy the following criteria. First, the amplitude is chosen lower than $10 \mathrm{kPa}$ and duration is longer than $1 \mathrm{~ms}$. These two conditions justify the omission of the convection term in the governing equations for air. Second, pulse energy is mostly distributed over frequencies higher than the critical frequency of the sample. This justifies the use of the high frequency formulation of complex compressibility function.

In order to show that Forchheimer's correction is important only for relatively high amplitude pulses, the data is

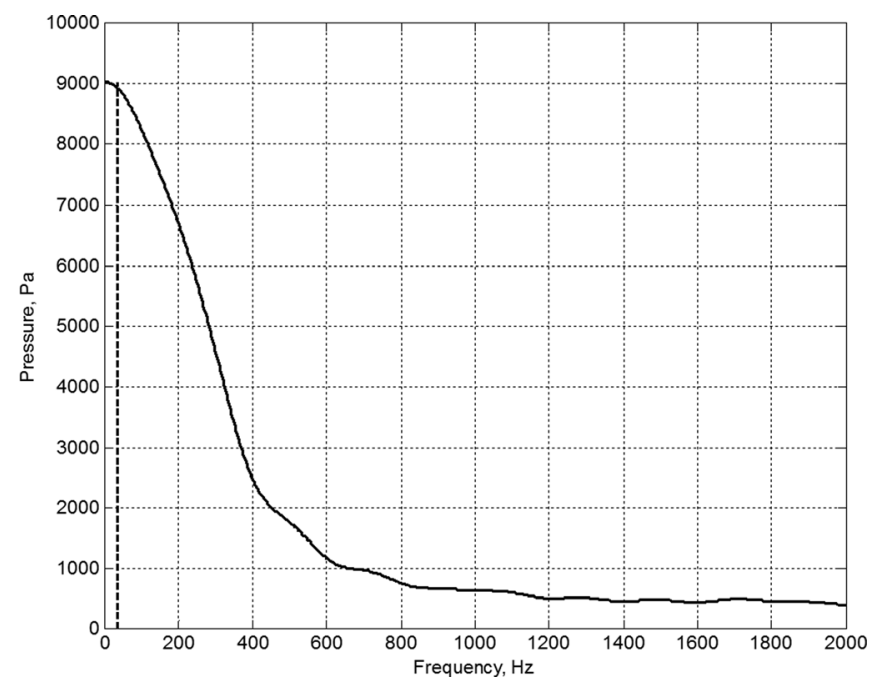

FIG. 7. Pulse spectrum of a high amplitude pulse $(3 \mathrm{kPa})$ measured with reflection setup. Dashed line: critical frequency of the lead shot. 
compared with predictions obtained (a) accounting for Forchheimer' correction and (b) without it, i.e., setting $\xi=0$ in Eq. (10).

The spectrum of the incident shock wave is shown in Fig. 7. It shows that more than $87 \%$ of the pulse energy is distributed above $f_{c}$.

The pressure peak of the pulse generated in a shock tube is initially higher than $10 \mathrm{kPa}$ (Fig. 6). However, after two reflections, the peak amplitude is reduced to $3 \mathrm{kPa}$. This pulse is used for comparisons with the model. The pulse recorded at Pos. 0, Fig. 4(a), is used as an input signal. The assumption that the amplitude and the duration do not change, when pulse travels a distance of $0.85 \mathrm{~m}$ to the surface of the sample, has been confirmed by numerical solution of the Burgess equation in air (24) as described in the previous section. It predicts less than $5 \%$ change in pulse amplitude and duration over this distance. For the transmission simulations, the pulse measured at Pos. 1, Fig. 4(b), is used as an input signal. However, the input signals shown in Fig. 8 is oversampled and reconstructed as a smoother function of time in order to eliminate the influence of the noise. It is shown in Fig. 8(b), that the transducer records superposition of the incident pulse and the pulse reflected from the sample. The waveform of the incident pulse used in the simulations is then obtained performing a linear interpolation

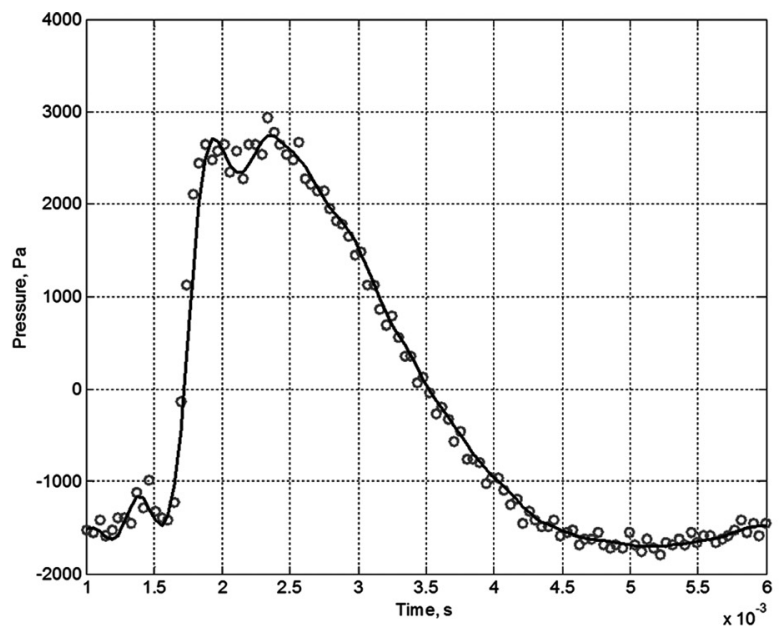

(a)

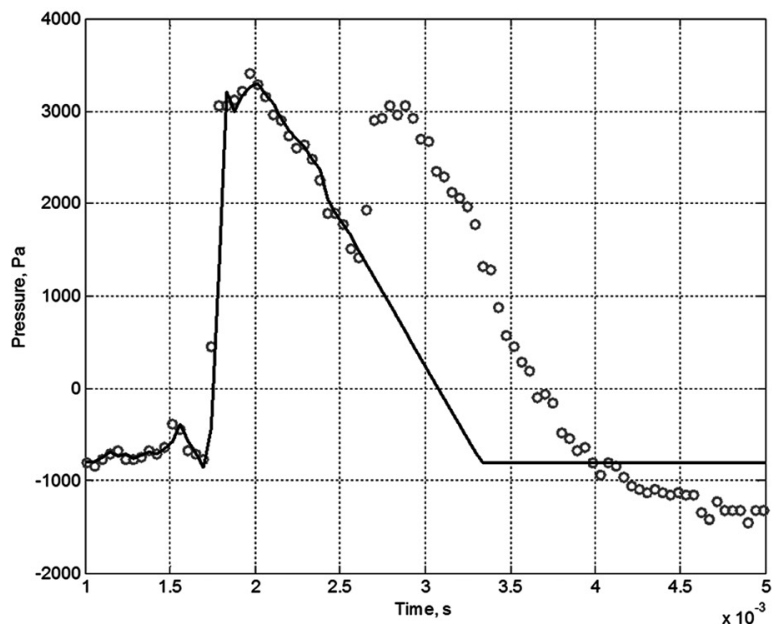

FIG. 8. Incident pulses used for reflection (a) and transmission (b) measurements. Gray circles: data; solid line: data reconstruction. and extrapolation of the data. Figures 9(a) and 9(b) show that accounting for Forchheimer's correction is crucial for correct predictions of both reflected pulse and pulse inside the rigidly backed material layer.

In order to investigate the range of pulse amplitudes and durations, where both transient and Forchheimer's nonlinearity effects are important, the following procedure is followed. First, it is assumed that the transient term is negligible and the following set of equations is solved numerically:

$$
\begin{aligned}
& \alpha_{\infty} \rho_{0} \frac{\partial v}{\partial t}+\sigma_{0} \phi(1+\xi|v|) v=-\phi \frac{\partial p}{\partial x} \\
& \frac{\partial p}{\partial t}+\frac{2(\gamma-1)}{\Lambda^{\prime}} \sqrt{\frac{\eta}{N_{\operatorname{Pr}} \pi \rho_{0}}} \int_{-\infty}^{t} \frac{\partial p}{\partial t^{\prime}} \frac{d t^{\prime}}{\sqrt{t-t^{\prime}}}=-\frac{\rho_{0} c_{0}^{2}}{\phi} \frac{\partial v}{\partial x}
\end{aligned}
$$

After that, the full system of equations (10) and (11) is solved. The comparisons of the predictions and the data obtained in a reflection set up are shown in Figs. 10(a) and 10(b). The effect
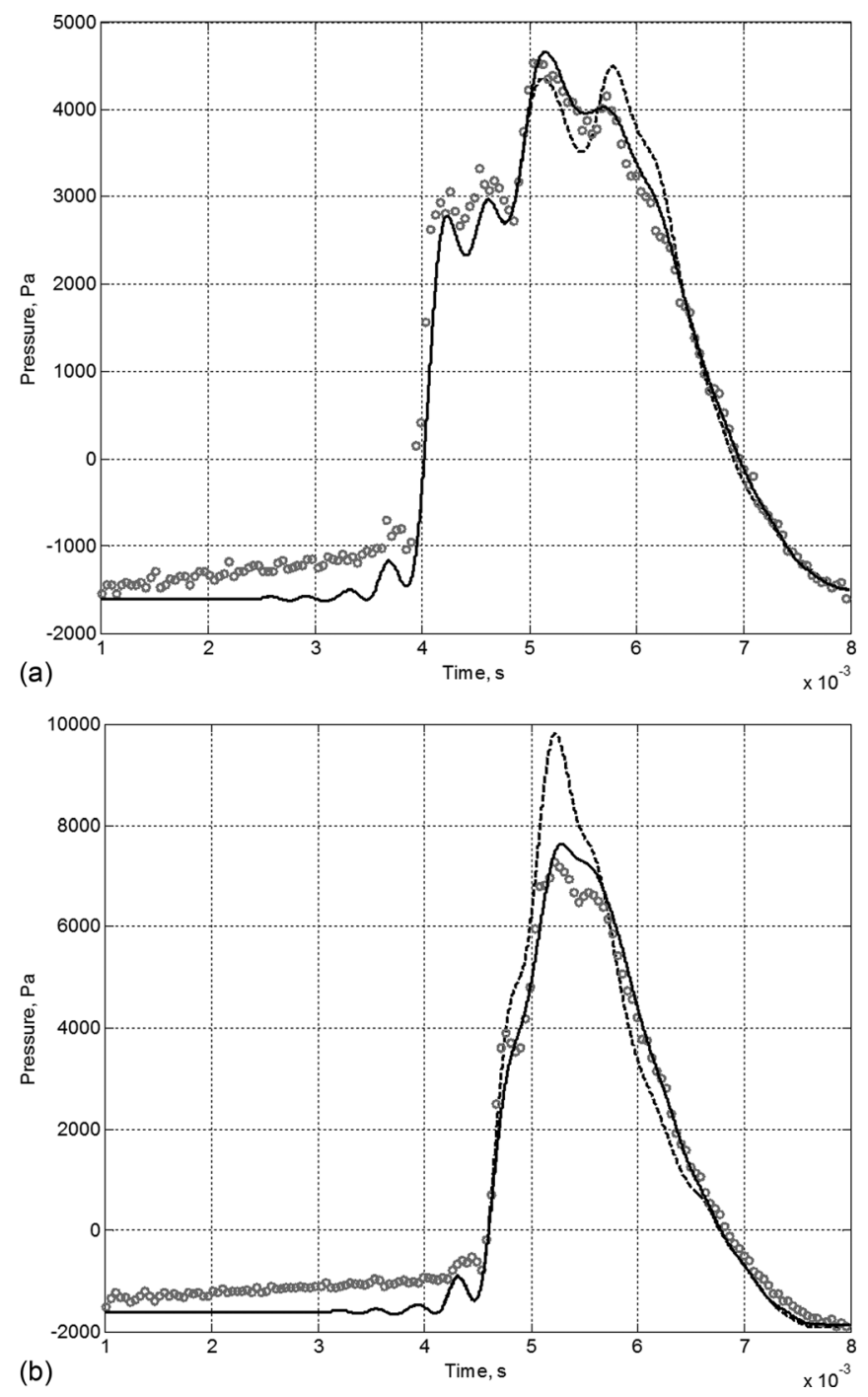

FIG. 9. Influence of the Forchheimer's nonlinearity. High amplitude pulse $(3 \mathrm{kPa})$, reflection set up. Lead shot layer, thickness $9 \mathrm{~cm}$. (a) Reflected pulse (Pos. 1). (b) Pulse recorded inside the layer (Pos. 2). Gray circles: data; solid line: full equations (10) and (11); dashed line: equations (10) and (11) without Forchheimer's nonlinearity term. 
of the transient term is weak, as predicted by the dimensionless analysis in the previous section. However, its inclusion gives a slightly better prediction of the reflected pulse, Fig. $10(\mathrm{a})$, and the pulse transmitted inside the porous sample, Fig. 10(b).

In Figs. 11(a)-11(c) the data obtained in a transmission set up are compared with the predictions. The influence of the transient effects on the reflected pulse [Fig. 11(a)] and on the one recorded inside the material [Fig. 11(b)] is weak. However, the extra attenuation introduced by the transient term improves the agreement between the data and the predictions for the pulse transmitted through the material layer, Fig. 11(c). The amplitude of the pulse traveling through the material is low, which makes the influence of the Forchheimer term weak and consequently increases the relative contribution of the transient term.

It can be concluded, that although in both low and high amplitude pulses the energy is mostly distributed in the range of frequencies above $f_{c}$, the transient effects are dominant for low amplitude pulse only, since in this case the
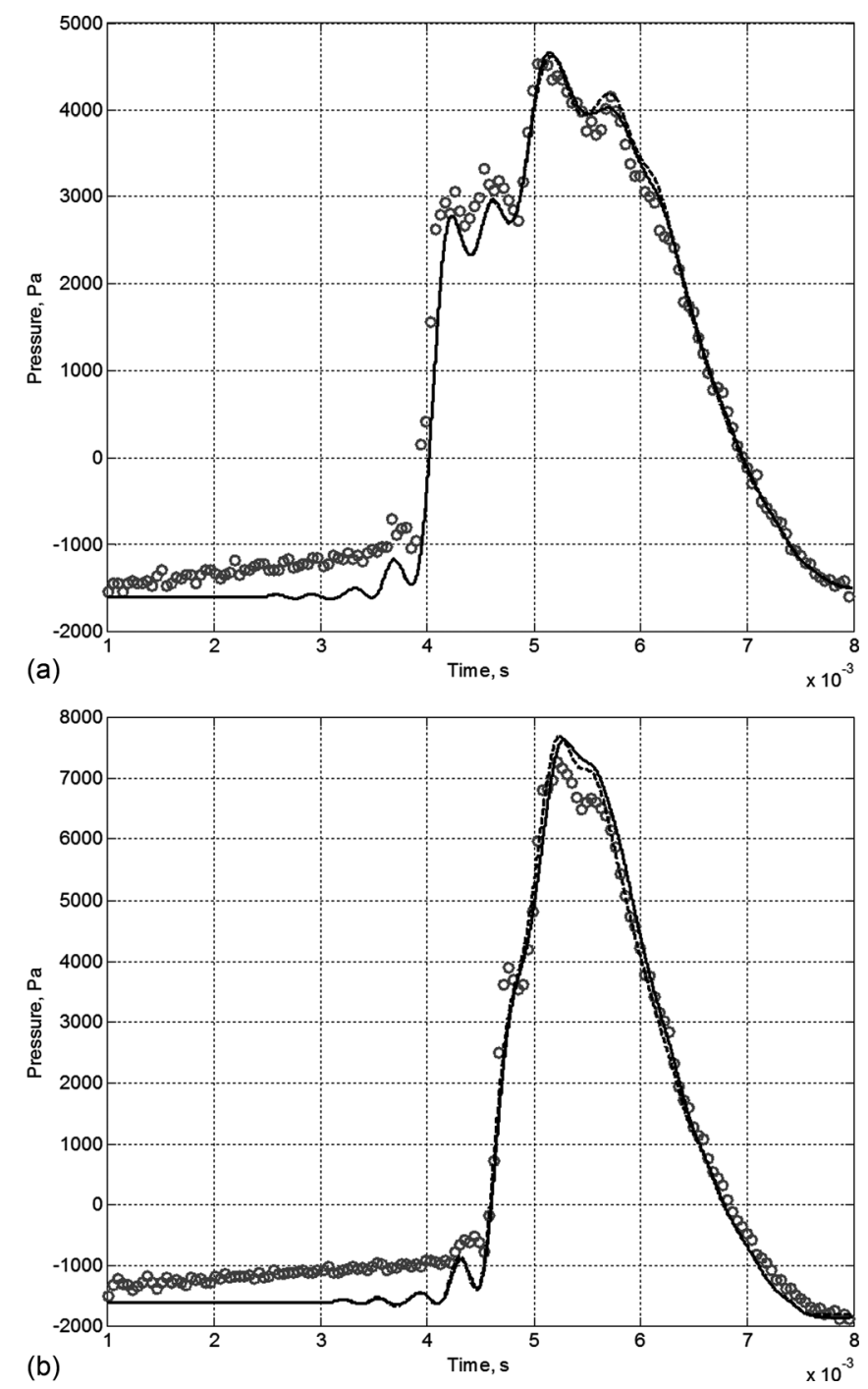

FIG. 10. Influence of the transient effects. High amplitude pulse $(3 \mathrm{kPa})$, reflection set up. Lead shot layer, thickness $9 \mathrm{~cm}$. (a) Reflected pulse (Pos. 1). (b) Pulse recorded inside the layer (Pos. 2). Gray circles: data; solid line: Eqs. (10) and (11); dashed line: Eqs. (26) and (27).
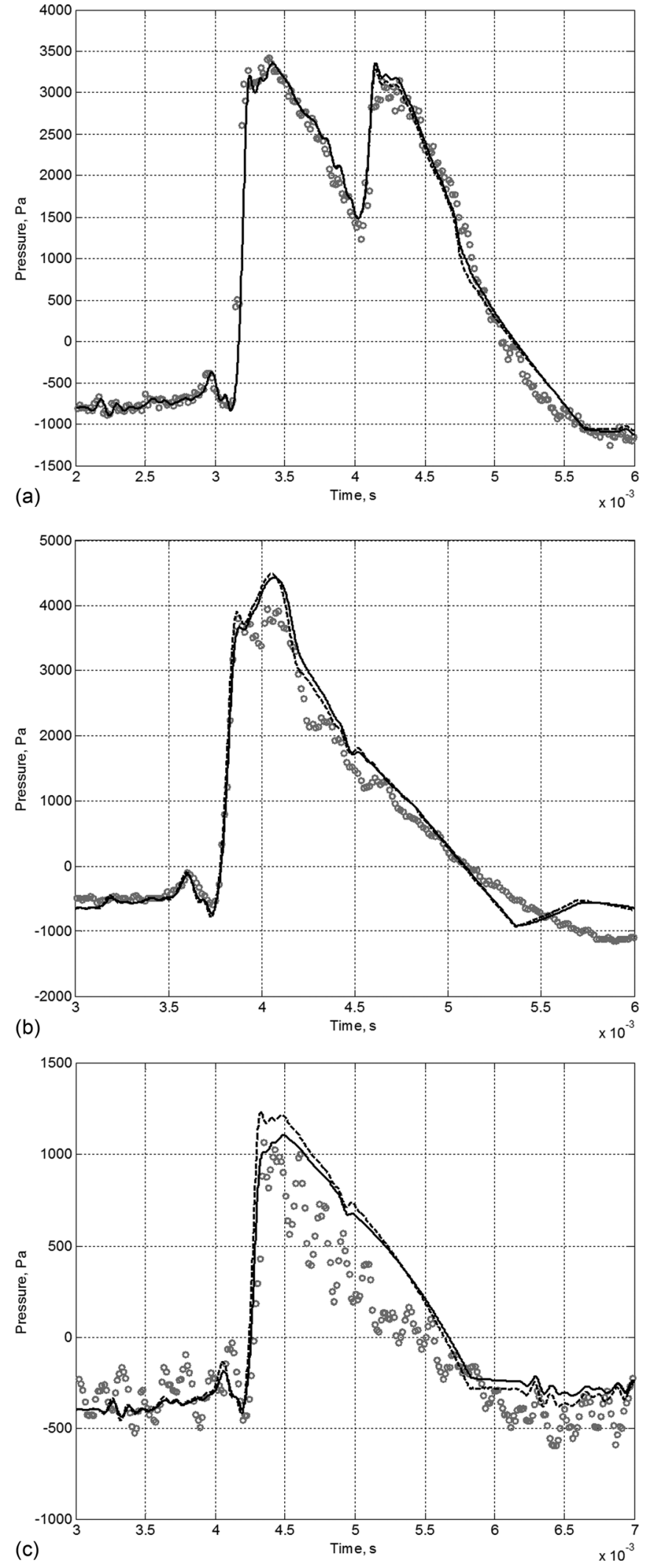

FIG. 11. Influence of transient effects. High amplitude pulse $(3.5 \mathrm{kPa})$, transmission set up. Lead shot layer, thickness $9 \mathrm{~cm}$. (a) Reflected pulse (Pos. 1). (b) Pulse recorded inside the layer (Pos. 2). (c) Transmitted pulse (Pos. 3). Gray circles: data; solid line: Eqs. (10) and (11); dashed line: Eqs. (26) and (27).

contribution of Forchheimer's correction is negligible. For higher amplitude pulse, the transient effects are weak compared to the Forchheimer's nonlinearity. 


\section{Reflection, transmission, and absorption coefficients at high levels of excitation}

In this section comparisons between the data and the model predictions for the acoustical characteristics of lead shot and gravel layers are presented. From pulse reflection and transmission measurements, described above, the reflection and transmission coefficients can be estimated. The reflection coefficient is defined as the ratio between the peak pressure of the reflected pulse and that of the incident one. The transmission coefficient is defined as the ratio of the peak pressure of the transmitted pulse and that of the incident pulse. It is also possible to estimate the attenuation coefficient using the pressure peak of the incident pulse $P_{\text {incident,peak }}$, at the porous material surface, and the pressure peak, $P_{\text {inside,peak }}$, recorded inside the porous medium at the position $d$. Then the attenuation coefficient $\alpha$ is evaluated as

$$
\alpha=-\frac{1}{d} \ln \left(\frac{P_{\text {inside,peak }}}{P_{\text {incident,peak }}}\right) \text {. }
$$
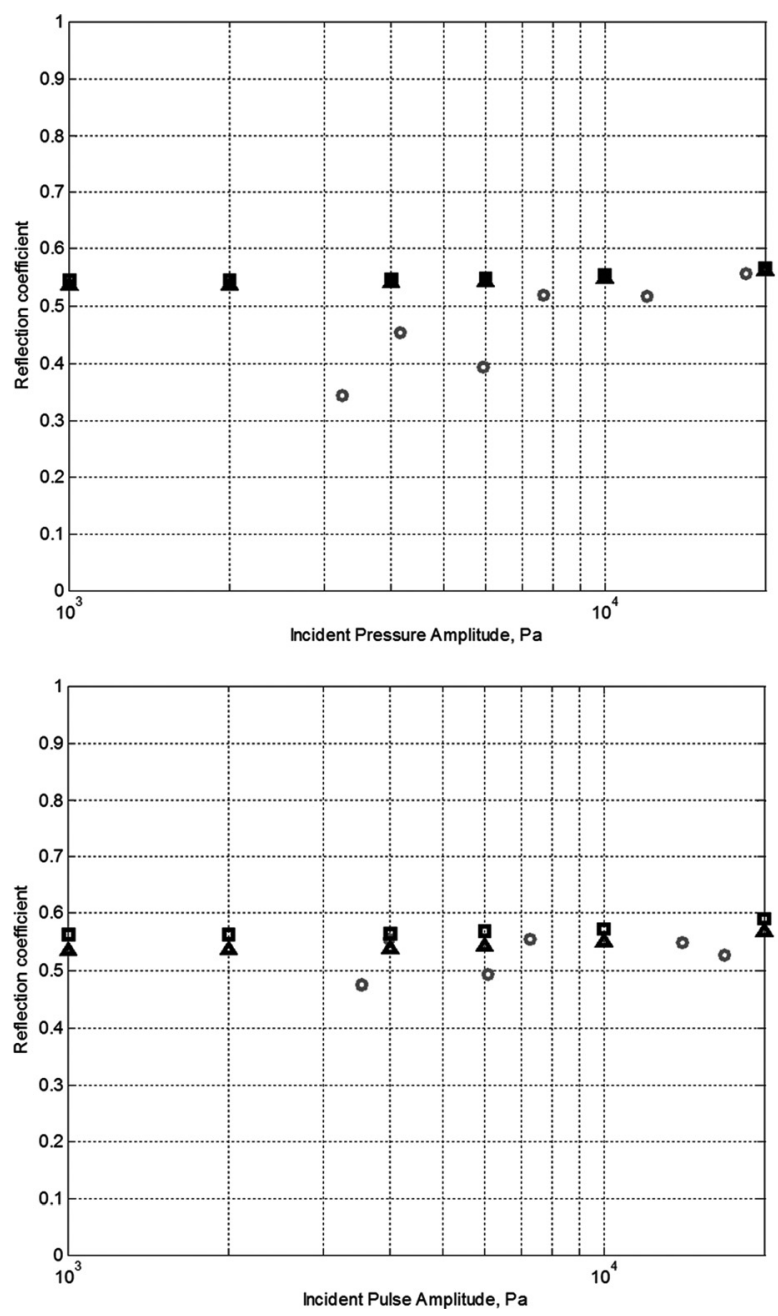

(a)

FIG. 12. Reflection coefficient dependence on the incident pulse amplitude. Reflection set up. (a) Lead shot (b) Gravel. Gray circle: data; black squares: numerical prediction using the model with both Forchheimer's nonlinearity and transient effects, Eqs. (10) and (11); black triangles: numerical prediction using the model with Forchheimer's nonlinearity only, Eqs. (26) and (27).
Using samples with thickness of $0.09 \mathrm{~mm}$, reflection, transmission, and attenuation coefficients are deduced for different pulse amplitudes.

A set of pulses with different amplitudes and durations is obtained performing a single foil rupture for each sample. When the pulse propagates inside the tube, its duration increases from 1 to $3 \mathrm{~ms}$ whereas the amplitude decreases from 20 to $2 \mathrm{kPa}$. However, a high proportion of the pulse energy is still distributed above the critical frequencies of the samples.

In order to investigate the importance of the transient effects, once again two different models are used for numerical simulations: one of them takes into account both Forchheimer nonlinearity and transient effects [Eqs. (10) and (11)] whereas the other takes into account Forchheimer's nonlinearity effect only [Eqs. (26) and (27)].

The dependence of reflection and transmission coefficients on the incident pulse amplitude is shown in Figs. 12(a) and 12(b) and Figs. 13(a) and 13(b).

The reflection coefficient increases with the increase of pulse amplitude, while the transmission coefficient decreases as the amplitude grows. This clearly demonstrates the nonlinear behavior. When the pulse amplitude grows, the flow resistivity of the medium grows as well due to Forchheimer's

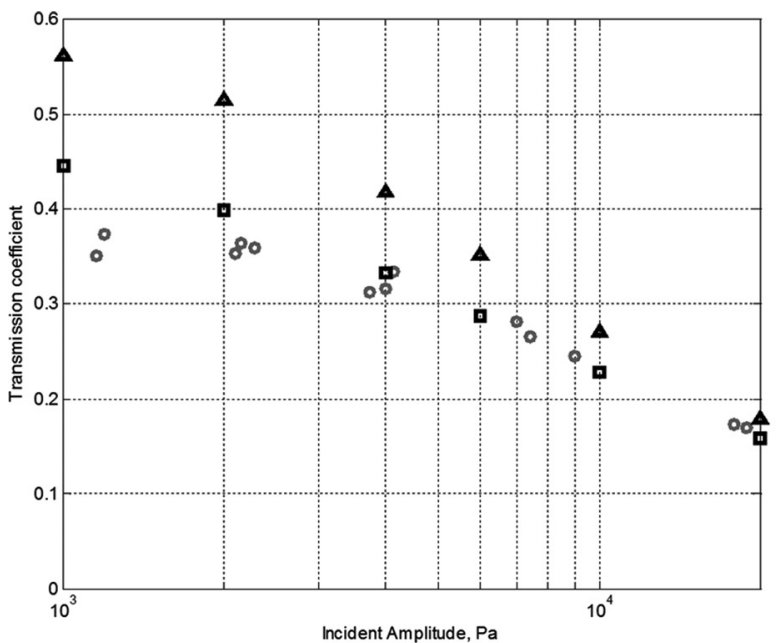

(a)

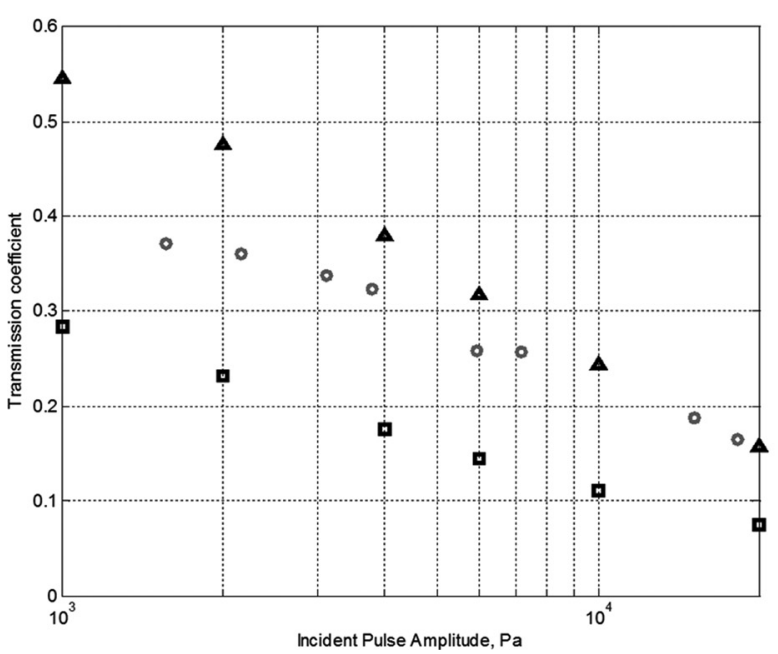

(b)

FIG. 13. Transmission coefficient dependence on incident pulse amplitude. Transmission set up. (a) Lead shot. (b) Gravel. Legend as in Fig. 12. 
nonlinearity. This increases the impedance mismatch between the air and the sample and leads to stronger reflections. The increase in losses inside the sample results in weaker transmission. For the lead shot sample the model is in a good agreement with the data. It should be noted that for lower pulse amplitudes, accounting for the transient effects improves the agreement between the model and the data [Fig. 13(a)]. For higher amplitudes, good agreement with the data is obtained for both models, confirming the relative weakness of the transient effects.

Attenuation coefficient dependence on the incident pulse amplitude is shown in Figs. 14(a) and 14(b). The higher is the pulse amplitude, the stronger is the attenuation inside the material. Again the agreement between the predictions of both models and the data is good for the lead shot and is less satisfactory for gravel. This disagreement could be due the fact that some parameters of the gravel are assumed to be equal to that reported in Ref. 13. They might not correspond to the particular packing used in measurements. In fact, gravel particles have irregular shape and their different packings can exhibit different acoustic properties.

For high amplitude pulses the predictions of both models (with and without transient effects) converge to the same limit where Forchheimer's nonlinearity is dominant and the
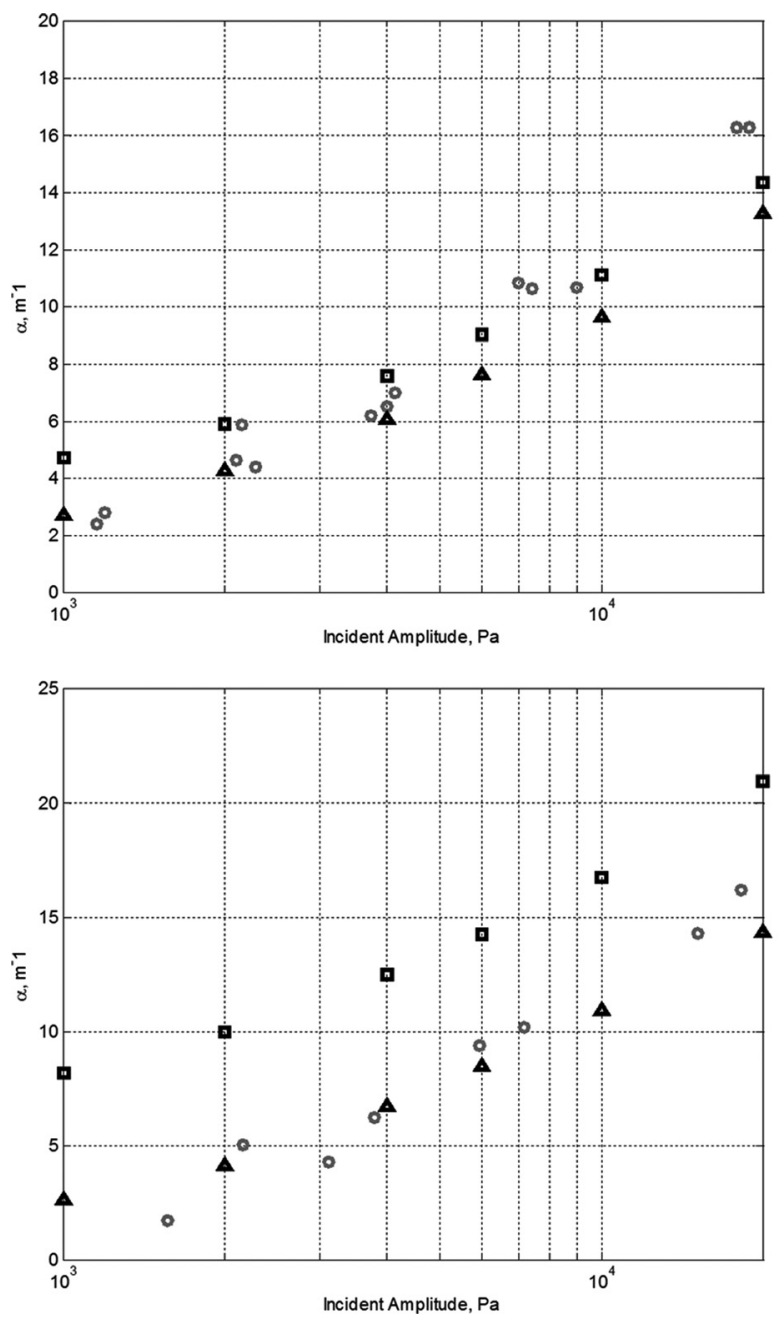

FIG. 14. Attenuation coefficient dependence on incident pulse amplitude. (a) Lead shot. (b) Gravel. Legend as in Fig. 12. transient effects contribution is insignificant. For the lower amplitudes, the transient effects become dominant and the predictions obtained accounting for it agree better with the data.

\section{CONCLUSIONS}

A time domain model describing finite amplitude pulse propagation in rigid porous materials has been developed. The model accounts for both Forchheimer's nonlinearity and transient effects. The approach is based on the assumption that the viscous and the transient term contributions to the drag force are additive. The model has been validated first in a linear regime by comparing its predictions with the reflection data for a low amplitude pulse. These measurements have been performed in an impedance tube using a layer of gravel. Further comparisons with the shock tube experiments have demonstrated that for a high amplitude pulse, reflection, and transmission data is in a satisfactory agreement with the model which accounts for the nonlinearity of material response.

For the materials considered in this work i.e. packings of relatively large lead shot and gravel, the transient effects dominate for low amplitude pulses with duration in the order of a millisecond. However, even for weak shocks with the same duration, the influence of transient effects is negligible compared to that of the Forchheimer's nonlinearity. It is shown, that due to strong Forchheimer's nonlinearity, the reflection coefficients of porous layers grow as pulse amplitude increases from 1 to $10 \mathrm{kPa}$, while the transmission coefficients decrease.

The dimensionless analysis suggests that in granular materials considered in this work and for the pulses with duration less than $0.01 \mathrm{~ms}$, the influence of the transient term might be significant even in the presence of Forchheimer's nonlinearity. However, considering the size of the particles (several millimeters), the high frequency content of such short pulses means that particles are no longer small compared to the wavelength and, consequently, sound propagates in a scattering regime.

The nonlinear model could be extended to include the effects of poro-elasticity. This is particularly important for high amplitude shocks when the sound induced compression is significant.

\section{ACKNOWLEDGMENT}

The work was supported by EPSRC Grant No. EP/E016529/1.

${ }^{1}$ S. Sorek, A. Levy, G. Ben-Dor, and D. Smeulders, "Contribution to theoretical/experimental developments in shock wave propagation in porous media," Transp. Porous Media 36, 63-100 (1999).

${ }^{2}$ O. Umnova, K. Attenborough, and A. Cummings, "High amplitude pulse propagation and reflection from a rigid porous layer," Noise Control Eng. 50, 204-210 (2002).

${ }^{3}$ Z. E. A. Fellah and C. Depollier, "Transient acoustic wave propagation in rigid porous media: A time domain approach,” J. Acoust. Soc. Am. 107, 683-688 (2000).

${ }^{4}$ Y. M. Shtemler, I. R. Shreiber, and A. Britan, "Shock induced flows through packed beds: Transient regimes," Acta Acust. 92, 612-617 (2006). 
${ }^{5} \mathrm{O}$. Umnova and D. Turo, "Time domain formulation of the equivalent fluid model for rigid porous media (L)," J. Acoust. Soc. Am. 125(4), 1860-1863 (2009).

${ }^{6}$ J. M. Evans and K. Attenborough, "Sound propagation in concentrated emulsions: Comparison of coupled phase model and core-shell model," J. Acoust. Soc. Am. 112(5), 1911-1917 (2002).

7L. D. Landau and E. M. Lifshitz, "Fluid Mechanics," in Course of Theoretical Physics, 2nd ed. (Pergamon, Oxford, 1959), Vol. 6, pp. 89-91, 388.

${ }^{8}$ Y. S. Lee and M. F. Hamilton, "Time-domain modelling of pulsed finiteamplitude sound beams," J. Acoust. Soc. Am. 97, 906-917 (1995).

${ }^{9}$ R. O. Cleveland, M. F. Hamilton, and D. T. Blackstock, "Time-domain modeling of finite-amplitude sound in relaxing fluid," J. Acoust. Soc. Am. 99, 3312-3318 (1996).

${ }^{10}$ D. L. Johnson, J. Koplik, and R. Dashen, "Theory of dynamic permeability and tortuosity in fluid-saturated porous media," J. Fluid. Mech. 176, 379-402 (1987).

${ }^{11}$ Y. Champoux and J.-F. Allard, "Dynamic tortuosity and bulk modulus in air-Saturated porous media," J. Appl. Phys. 70, 1975-1979 (1991).

${ }^{12}$ D. Lafarge, P. Lemariner, J.-F. Allard, and V. Tarnow, "Dynamic compressibility of air in porous structures at audible frequencies," J. Acoust. Soc. Am. 102, 1995-2006 (1997).

${ }^{13}$ O. Umnova, K. Attenborough, E. Standley, and A. Cummings, "Behavior of rigid-porous layers at high levels of continuous acoustic excitation: Theory and experiment," J. Acoust. Soc. Am. 114(3), 1346-1356 (2003).

${ }^{14}$ O. Umnova, K. Attenborough, H.-C. Shin, and A. Cummings, "Response of multiple rigid porous layers to high levels of continuous acoustic excitation,” J. Acoust. Soc. Am. 116, 703-712 (2004).

${ }^{15}$ O. Umnova, K. Attenborough, and K. M. Li, "A cell model for the acoustical properties of packings of spheres," Acta Acust. 87, 226-235 (2001).

${ }^{16}$ D. K. Wilson, V. E. Ostashev, S. L. Collier, N. P. Symons, D. F. Aldridge, and D. H. Marlin, "Time-domain calculations of sound interactions with outdoor ground surfaces," Appl. Acoust. 68, 173-200 (2007).

${ }^{17}$ W. F. Ames, Numerical Methods for Partial Differential Equations, 3rd ed. (Academic, New York, 1992), pp. 165-229.

${ }^{18} \mathrm{D}$. Turo and O. Umnova, "Time domain modelling of sound propagation in porous media and the role of shape factors," Acta Acust. 96, 225-238 (2010).

${ }^{19}$ E. Standley, O. Umnova, K. Attenborough, A. Cummings, and P. Dutta, "Shock wave reflection measurements on porous materials," Noise Control Eng. J. 50(6), 224-230 (2002).

${ }^{20} \mathrm{~K}$. Naugolnykh and L. Ostrovsky, Nonlinear Wave Processes in Acoustics (Cambridge University Press, Cambridge, 1998), pp. 7-9.

${ }^{21}$ C. A. J. Fletcher, Computational Techniques for Fluid Dynamics 2. Specific Techniques for Different Flow Categories, 2nd ed. (SpringerVerlag, Berlin, 2006), pp. 144-147. 